\title{
APRENDER A CONSTRUIR COM TERRA ATRAVÉS DA ANDORINHA-DOS-BEIRAIS
}

\author{
Bruno Silva* (Portugal) \\ Departamento de Engenharias, ECT. Universidade de Trás-os-Montes e Alto Douro (UTAD) \\ Fernando Nunes** (Portugal) \\ CQVR. Universidade de Trás-Os-Montes e Alto Douro (UTAD) \\ José Correia*** (Portugal) \\ CQVR. Universidade de Trás-Os-Montes e Alto Douro (UTAD) \\ Pedro Tavares**** (Portugal) \\ CQVR. Universidade de Trás-Os-Montes e Alto Douro (UTAD) \\ Humberto Varum ${ }^{\dagger}$ (Portugal) \\ Departamento de Engenharia Civil. Universidade de Aveiro (UA) \\ Jorge Pinto ${ }^{\ddagger}$ (Portugal) \\ Departamento de Engenharias, ECT. Universidade de Trás-Os-Montes e Alto Douro (UTAD)
}

\section{RESUMO}

O facto de a terra ser natural, ecológica, reciclável e abundante faz com que possa ser considerada um material de construção de eleição e com um enorme potencial. Na construção tradicional portuguesa as soluções construtivas que usam terra como material de construção têm uma importante expressão. Este património construído necessita de ser conservado. Neste contexto, o presente trabalho tem como principal objectivo dar um contributo na temática das soluções de melhoramento do comportamento e do reforço de elementos construtivos de terra a partir do estudo biomimético de um ninho da andorinha-dosbeirais. De modo a identificar a eventual existência de algum fenómeno de aglutinação ocorrido durante o processo de construção do ninho da andorinha-dos-beirais, procedeu-se a um trabalho experimental de caracterização a partir de amostras extraídas de diferentes ninhos recolhidos na zona de Vila Real. Para a identificação/caracterização da composição química inorgânica elementar e mineralógica realizaramse análises em Scanning Electron Microscopy/Energy Dispersive Spectroscopy (SEM/EDS) e em difracção de raios-X. Para a identificação/caracterização da composição orgânica foi feita a quantificação colorimétrica do teor de proteínas pelo método do biureto, e do teor de polissacarídeos/açucares pelo método dos açúcares totais. Os resultados experimentais apontam para a existência de polissacarídeos/ açucares no material do ninho e que possívelmente foram adicionados pela andorinha-dos-beirais. Por sua vez, uma simulação numérica de um ninho permitiu compreender o funcionamento desta estrutura natural de terra e verificando-se que ele funciona essencialmente à compressão. Ensaios mecânicos de provetes de solo argiloso misturado com uma solução aquosa à base de um polímero extraído do ninho revelaram uma melhoria de comportamento mecânico. Pretende-se que a partir dos resultados obtidos neste trabalho de investigação se possam retirar sugestões para o desenvolvimento de soluções de estabilização e de melhoramento de elementos construtivos existentes de terra.

Palavras-chave: Construção de terra, construção sustentável, materiais naturais.

*bruno_silva06@hotmail.com

**fnunes@utad.pt

***jcorreia@utad.pt

****ptavares@utad.pt

thvarum@ua.pt

$\ddagger_{\text {tiago@utad.pt }}$ 


\section{INTRODUÇÃO}

Cerca de metade da população mundial, aproximadamente três bilhões de pessoas em seis continentes, habita ou trabalha em edifícios construídos em terra (Rael, 2008). Nos países menos desenvolvidos esta realidade atinge cerca de metade dos edifícios. A construção de terra é um património mundial que reflecte a diversidade cultural (Carvalho et al., 2008).

Exemplos de construções antigas de terra são a Muralha da China, na China, de 2000 A.C. (Greatwallchina, 2005) e a cidade de Arge Bam, no Irão, de 500 a.C, este impressionante património de terra também é revelador que este tipo de construção pode ter uma enorme durabilidade. Paralelamente, também existem construções de terra de vanguarda tais como o Adobe Repository for Buda Statue, no Japão, de 2001 (Rael, 2008) e o Desert Cultural Centre, no Canada, de 2006 e que são reveladoras de que a construção de terra é uma alternativa de construção do futuro.

A biomimética é uma área da ciência que se baseia no estudo da natureza de forma a extrapolar novas formas de adaptação ao planeta. Mecanismos biológicos e estruturas naturais são dois exemplos de modelos naturais de imitação usados na biomimética. Têm sido realizados inúmeros trabalhos de investigação nesta área científica e em campos de aplicação diversos (Bar-Cohen, 2006). São exemplos, os mecanismos e as estratégias de defesa/ataque inspirados em organismos vivos, trabalhos sobre nano-materiais e transplantes de órgãos humanos e o conceito de pré-esforço orgânico inspirado no modelo do músculo do bíceps humano (André et al., 2003).

No presente trabalho, o ninho da andorinha-dos-beirais foi o modelo natural estudado. Outros exemplos de construções naturais à base de terra são o ninho da vespa solitária e o ninho das térmitas. Este trabalho de investigação pretendeu aferir se a andorinha-dos-beirais introduz alguma espécie de aglutinante natural na fase de construção do ninho e que possa induzir uma melhoria de qualidade do material. Esta informação poderá ser extrapolada para o contexto da construção civil e, em particular, para a construção de terra.

Para o efeito, foi necessário e fundamental efectuar uma identificação/caracterização exaustiva do material constituinte dos ninhos em termos de composição química elementar, de composição mineralógica e de composição orgânica, através de um estudo experimental que incluiu análises em Scanning Electron Microscopy/ Energy Dispersive Spectroscopy (SEM/EDS), difracção de raios- $X$, análises colorimétricas e cromatografia iónica. Os resultados experimentais obtidos indicam que a andorinha-dos-beiriais aparentemente adiciona uma pequena quantidade de polissacarídeoas/açucares ao solo argiloso durante o processo de construção do ninho.

Uma simulação numérica do ninho permitiu verificar que esta estrutura natural trabalha essencialmente à compressão e que é uma característica inerente às construções de terra. Por fim, também foram realizados ensaios de compressão em provetes de solo argiloso misturado com uma solução aquosa de polissacarídeoas/ açucares extraída do material do ninho que revelaram um aumento da resistência à compressão.

\section{A ANDORINHA-DOS-BEIRAIS}

A andorinha-dos-beirais, figura 1, é uma ave migratória. Em média, o seu comprimento é de 12,5 cm e seu peso é de 18,3 $\mathrm{g}$. Cada postura inclui quatro ou cinco ovos $11,9 \mathrm{~cm}$ comprimento, $1,3 \mathrm{~cm}$ de diâmetro e 1,7 g de peso). Geralmente, há duas posturas por um período estival. O ninho que é uma estrutura natural feita de solo argiloso recolhido em cursos de água existentes em zonas circundantes e próximas do ninho, é geralmente reutilizado em anos consecutivos o que requer que a andorinhados-beirais tenha que o reparar.

Entre outros países da Europa, esta ave também passa o período estival em Portugal e, em particular, na cidade de Vila Real (capital da região de Trás-os- 


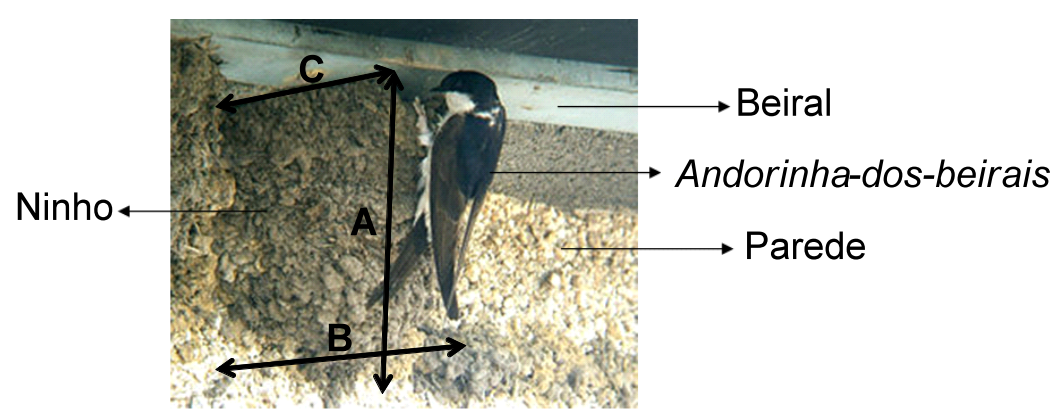

Fig. 1. Andorinha-dos-beirais e ninho

Montes e Alto Douro, parte nordeste de Portugal continental) onde este trabalho de investigação foi desenvolvido.

\section{O NINHO DA ANDORINHA-DOS-BEIRAIS}

Actualmente é muito frequente encontrar a andorinha-dos-beirais nas áreas urbanas e em zonas próximas de cursos de água. $\mathrm{Na}$ figura 1 é mostrado um ninho da andorinhados-beirais, este é construído na junção da parede e do beiral permitindo desta forma a existência de duas superfícies de contacto para o suporte do ninho e criar condições de protecção do ninho em relação à chuva e ao ataque de predadores. Pode-se também observar na Figura 1 que a parede do ninho é formada por pequenas porções de argila ligadas entre si e á semelhança de uma construção de adobe. Observa-se também que as dimensões das porções de argila tendem a diminuir no sentido da base para o topo do ninho.

Neste trabalho de investigação foram usadas seis amostras de ninho que foram recolhidas em três locais diferentes de Vila Real e que são São Dinis, Mateus e Noura, figura 2. As dimensões médias de um ninho da andorinha-dos-beirais são $12 \mathrm{~cm}$ de altura (A), $18 \mathrm{~cm}$ de largura (B), $8 \mathrm{~cm}$ de profundidade (C) e $2 \mathrm{~cm}$ de espessura de parede.

Nos ensaios de identificação/ caracterização do material do ninho, que serão apresentados e discutidos nas secções seguintes, foram considerados

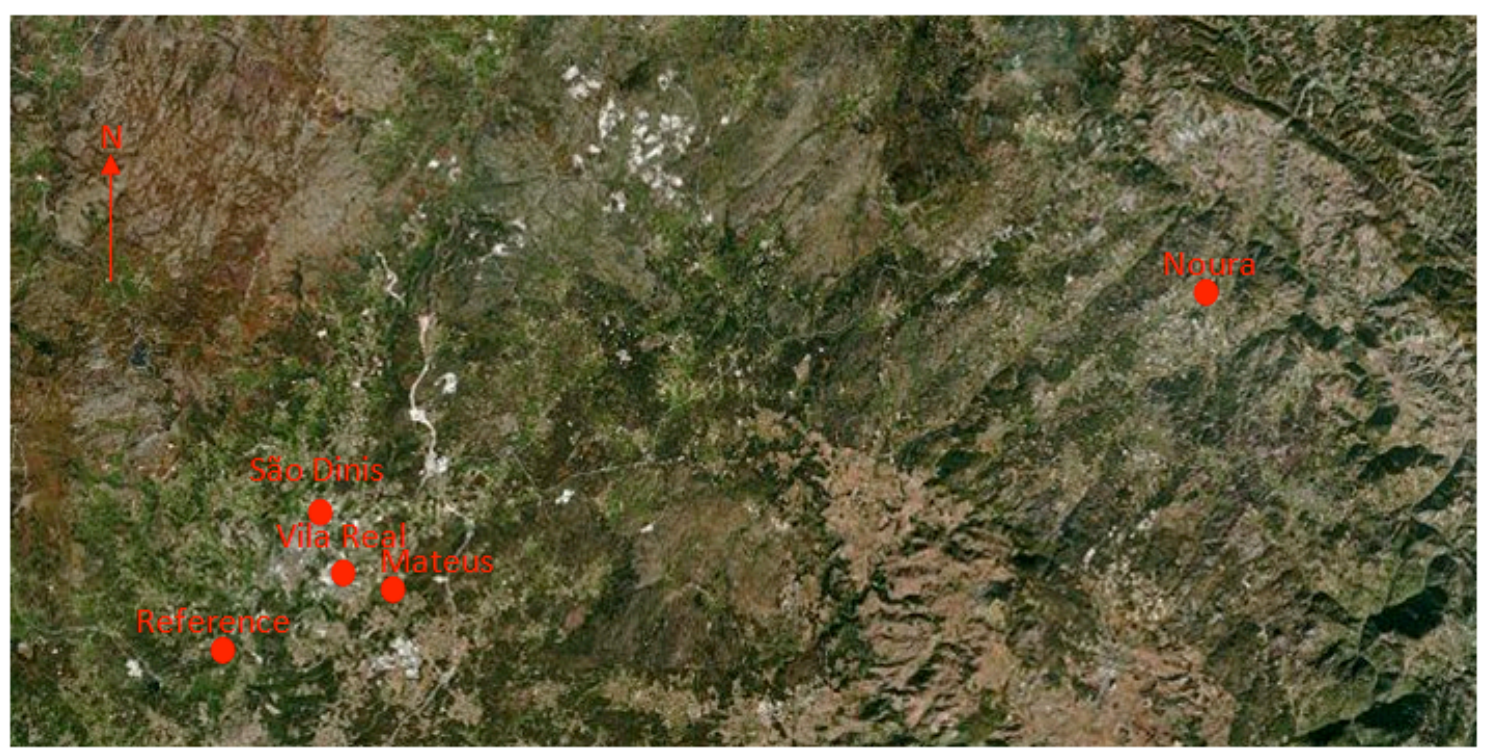

Fig. 2. Locais de recolha do material de referência e dos ninhos (Fonte: http://maps.google.pt/maps?hl=ptPT\&tab=wl) 
três tipos de amostras de ninho e que são relativas aos três locais de recolha (São Dinis, Mateus e Noura). A amostra por local de recolha foi obtida através da mistura do material dos dois ninhos recolhidos nesse local.

\section{COMPORTAMENTO ESTRUTURAL DO NINHO}

De modo a melhor se perceber o comportamento estrutural do ninho foi feita a sua simulação numérica através de um modelo de elementos finitos em 3D, usando o código comercial ANSYS ${ }^{\circledR}$ (ANSYS, 2008). Foi desenvolvido um modelo

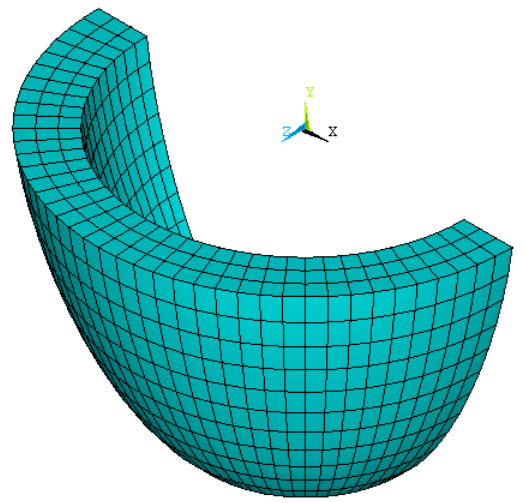

a) Malha de elementos finitos

Fig. 3. Modelo numérico do ninho

paramétrico em linguagem APDL do ANSYS ${ }^{\circledR}$, considerando para tal, elementos do tipo sólido isoparamétricos hexaédricos de 20 nós (SOLID95). A figura 3-a ilustra a malha de elementos finitos resultante em que a abertura existente no topo do ninho não foi considerada por simplificação. O material do ninho foi considerado isotrópico, elástico, com um peso específico de 18 kN/m3, com um módulo de elasticidade de $1 \mathrm{GPa}$ e com um coeficiente de Poisson de 0,25. Os deslocamentos dos nós que simulam o contacto ninho/parede e ninho/padieira foram restringidos em todas as direcções.

O diagrama de tensões normais segundo a direcção vertical (y) apresentado na figura 3-b é relativo ao caso de carga mais gravoso (peso próprio do ninho e peso de seis aves adultas). Esse diagrama de tensões mostra que o ninho está essencialmente sujeito a tensões normais de compressão sendo apenas excepção as zonas pontuais do contacto ninho/beiral e na face interna do ninho.

\section{IDENTIFICAÇÃO/CARACTERIZAÇÃO DO MATERIAL DO NINHO}

Tal como foi referido anteriormente, o ninho da andorinha-dos-beirais é construído à base de um solo argiloso. De forma a verificar este facto e a verificar se a andorinha-dos-beirais incorpora algum componente ao solo durante o processo

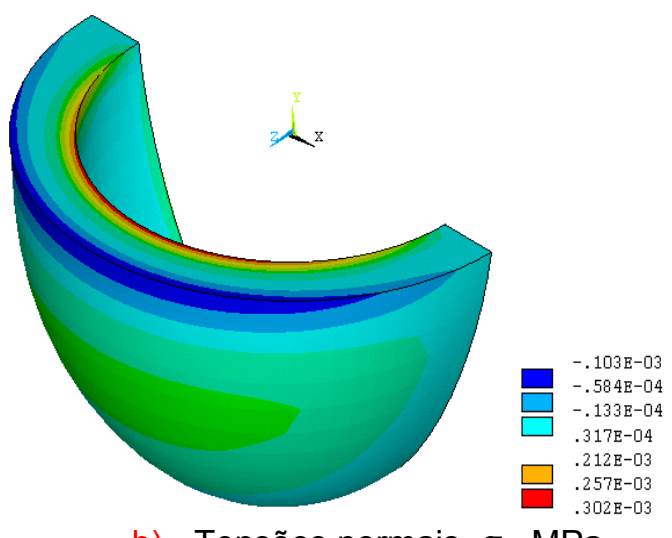

b) Tensões normais, $\sigma_{y}, \mathrm{MPa}$

de construção do ninho foram realizados vários ensaios tais como SEM/EDS, difracção de raios- $X$, análise colorimétrica e cromatografia iónica.

A apresentação e a análise dos resultados experimentais relativos aos ensaios de SEM/EDS, difracção de raios-X e análise colorimétrica já foram apresentados em (Bruno et al., 2009) onde se concluiu que os ensaios de SEM/EDS e de difracção de raios-X mostraram que as composições química elementar e mineralógica do material do ninho são similares às do solo argiloso local e, que as análises colorimétricas revelaram que existe uma quantidade expressiva de proteínas no material de ninho. Por outro lado, estas análises permitiram concluir que existe a possibilidade da andorinha-dos-beirais adicionar uma pequena quantidade 
de componentes orgânicos do tipo polissacarídeos/açúcares no solo argiloso durante o processo de construção do ninho.

De forma a identificar/caracterizar os tipos de polissacarídeos/açúcares e a quantificar estes componentes foram realizadas análises de cromatografia iónica, no laboratório de Cromatografia, da UTAD. Os polissacarídeos/açúcares foram extraídos das amostras através de hidrólise directa. Os tipos de polissacarídeos/ açúcares estudados são aqueles que são considerados os mais comuns na natureza (Lee, 1996) e que são a fucose (Fuc), a arabinose (Ara), a galactose (Gal), a glucosamina (GlcN), a glicose (Glc), a manose (Man) e a xilose (Xyl).

Os resultados estão apresentados na tabela 1 verificando-se que todas as amostras de materiais incluindo a amostra de Referência, têm polissacarídeos/açúcares nas suas composições. O material das amostras de ninho de Mateus e de São Dinis têm uma percentagem de polissacarídeos/açúcares superior (35\% e $33 \%$, respectivamente) às amostras de material do ninho de Noura e de Referência (13\% e 19\%, respectivamente).

Em termos de quantidade de polissacarídeos/açúcares, a amostra do ninho Noura é aquela que é mais semelhante à amostra de Referência. Este resultado talvez possa estar relacionado com o facto do ninho de Noura ter sido recolhido num dia chuvoso e ter, por isso, havido um lixíviamento de material.

Considerando a possibilidade da andorinhados-beirais adicionar uma certa quantidade de polissacarídeos/açúcares ao material durante o processo de construção do ninho, será seguidamente identificado o tipo de polissacarídeos/açúcar que existe em maior quantidade nas amostras de ninho em relação à amostra de Referência. Os resultados da tabela 1 indicam que esse tipo de polissacarídeo/açúcar poderá ser a Glc porque as amostras de material do ninho de Mateus e de Noura apresentam $42 \%$ e $44 \%$ de Glc, respectivamente, enquanto que o respectivo valor da amostra de Referência é de $40 \%$.

Com base nestes resultados, pode-se pensar que existe a possibilidade da andorinhados-beirais adicionar uma pequena quantidade (aproximadamente 3\%) de Glc no material do ninho durante o seu processo de construção.

Contudo, estes resultados também evidenciam alguma discrepância e que poderá estar relacionada com o facto do solo argiloso utilizado como amostra de Referência não ser representativo do solo argiloso existente na região. Um trabalho de investigação adicional necessita de ser desenvolvido nesta matéria utilizando para o efeito um maior número de amostras de solo e também de ninhos.

\begin{tabular}{|c|c|c|c|c|c|c|c|}
\hline Amostras & $\begin{array}{c}\text { Fuc } \\
(\mathrm{mg} / \mathrm{g})\end{array}$ & $\begin{array}{c}\text { Ara } \\
(\mathrm{mg} / \mathrm{g})\end{array}$ & $\begin{array}{c}\text { Gal } \\
(\mathrm{mg} / \mathrm{g})\end{array}$ & $\begin{array}{c}\text { GlcN } \\
(\mathrm{mg} / \mathrm{g})\end{array}$ & $\begin{array}{c}\text { Glc } \\
(\mathrm{mg} / \mathrm{g})\end{array}$ & $\begin{array}{c}\text { Man/Xyl } \\
(\mathrm{mg} / \mathrm{g})\end{array}$ & $\begin{array}{l}\text { Total_1 } \\
(\mathrm{mg} / \overline{\mathrm{g}})\end{array}$ \\
\hline Referência & $\begin{array}{c}0,014 \\
(1.7 \%)\end{array}$ & $\begin{array}{c}0,177 \\
(21.5 \%)\end{array}$ & $\begin{array}{l}0,174 \\
(21 \%)\end{array}$ & $\begin{array}{l}0,00 \\
(0 \%)\end{array}$ & $\begin{array}{l}0,332 \\
(40 \%)\end{array}$ & $\begin{array}{l}0,128 \\
(16 \%)\end{array}$ & $\begin{array}{l}0,825 \\
(19 \%)\end{array}$ \\
\hline Mateus & $\begin{array}{l}0,017 \\
(1 \%)\end{array}$ & $\begin{array}{l}0,220 \\
(15 \%)\end{array}$ & $\begin{array}{c}0,156 \\
(11 \%)\end{array}$ & $\begin{array}{c}0,257 \\
(17 \%)\end{array}$ & $\begin{array}{l}0,614 \\
(42 \%)\end{array}$ & $\begin{array}{l}0,208 \\
(14 \%)\end{array}$ & $\begin{array}{l}1,472 \\
(35 \%)\end{array}$ \\
\hline Noura & $\begin{array}{l}0,020 \\
(3 \%)\end{array}$ & $\begin{array}{l}0,116 \\
(20 \%)\end{array}$ & $\begin{array}{l}0,105 \\
(10 \%)\end{array}$ & $\begin{array}{l}0,00 \\
(0 \%)\end{array}$ & $\begin{array}{l}0,248 \\
(44 \%)\end{array}$ & $\begin{array}{l}0,077 \\
(14 \%)\end{array}$ & $\begin{array}{l}0,565 \\
(13 \%)\end{array}$ \\
\hline São Dinis & $\begin{array}{l}0,013 \\
(1 \%)\end{array}$ & $\begin{array}{l}0,202 \\
(15 \%)\end{array}$ & $\begin{array}{l}0,571 \\
(42 \%)\end{array}$ & $\begin{array}{l}0,00 \\
(0 \%)\end{array}$ & $\begin{array}{l}0,427 \\
(30 \%)\end{array}$ & $\begin{array}{l}0,165 \\
(12 \%)\end{array}$ & $\begin{array}{l}1,379 \\
(33 \%)\end{array}$ \\
\hline & & & & & & $\begin{array}{c}\text { Total_2 } \\
(\mathrm{mg} / \mathrm{g})\end{array}$ & 4,241 \\
\hline
\end{tabular}

Tabela 1. Concentração de polissacarídeos/açúcares 


\section{ENSAIOS DE COMPRESSÃO}

De forma a verificar se a adição dos polissacarídeos/açúcares identificados anteriormente no solo argiloso se traduz numa melhoria das propriedades mecânicas do material e em especial na resistência à compressão, ensaios de compressão foram realizados no laboratório de Materiais e Solos, da UTAD. Para o efeito, foram fabricados dois tipos de provetes usando solo argiloso recolhido no curso de água identificado anteriormente, figura 2. O primeiro tipo de provetes (tipo I) foi fabricado misturando ao solo argiloso 50 cl de água ultra pura. O segundo tipo de provetes (tipo II) foi fabricado misturando ao solo argiloso $50 \mathrm{cl}$ de uma solução aquosa extraída do material do ninho.

O processo de extracção dos polissacarídeos/açúcares do material do ninho consistiu essencialmente em provetes foram ensaiados à compressão, à idade de setes dias, à temperatura ambiente do laboratório e usando o equipamento Machine 5equipment BETA. Os resultados obtidos através do ensaio de compressão estão apresentados na tabela 2 e indicam que os provetes tipo II têm uma maior resistência à compressão do que os provetes tipo I o que sugere que a inclusão de polissacarídeos/açúcares no solo argiloso poderá aumentar as propriedades mecânicas de material. Esta conclusão poderá ser de grande interesse para a melhoria do desempenho estrutural de construções de terra.

Os valores de resistência à compressão obtidos nos provetes tipo I apresentam uma grande dispersão. A justificação para este facto só poderá ser encontrada através da realização de novos ensaios à compressão usando um maior número de provestes e um maior rigor na preparação do material

\begin{tabular}{cccccc}
\hline \multicolumn{7}{c}{ Provetes } \\
\hline $\mathrm{I}-1$ & $\mathrm{I}-2$ & $\mathrm{I}-3$ & $\mathrm{II}-1$ & $\mathrm{II}-2$ & $\mathrm{II}-3$ \\
\hline 0,27 & 0,65 & 0,49 & 0,71 & 0,68 & 0,76 \\
\hline & Média (Tipo I): $0,47 \mathrm{MPa}$ & \multicolumn{3}{c}{ Média (Tipo II): 0,72 MPA } \\
\hline
\end{tabular}

Tabela 2. Resultados dos ensaios de compressão, MPa

três etapas. Inicialmente, as amostras foram limpas e trituradas. Em seguida, os componentes orgânicos das amostras de material do ninho foram extraídos misturando-se $250 \mathrm{~g}$ de material do ninho triturado com $50 \mathrm{cl}$ de água ultra pura. Finalmente, a solução aquosa extraída foi filtrada.

Foram fabricados três provetes cúbicos de $4 \times 4 \times 4 \mathrm{~cm} 3$ para cada tipo I e II. Esses

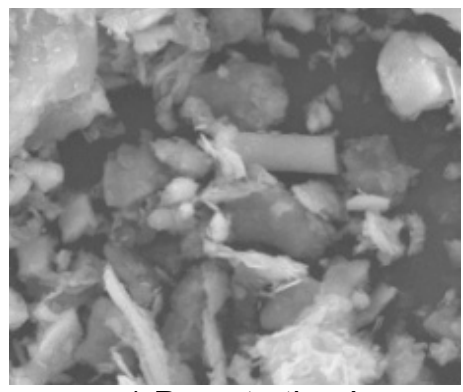

a) Provete tipo I terra a ensaiar. Um trabalho de investigação adicional está em curso para este efeito.

As imagens microscópicas de provetes tipo I e II apresentadas na figura 4 também sugerem que existe uma diferença morfológica acentuada nos dois tipos de provetes e talvez seja resultante de um possível processo de aglutinação resultante da adição de polissacarídeos/açúcares ao solo argiloso (provetes tipo II).

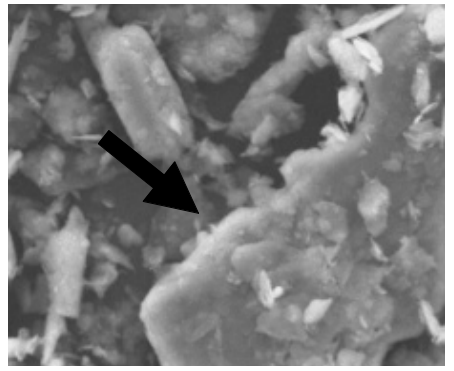

b) Provete tipo II

Fig. 4. Imagens microscópicas 


\section{PRINCIPAIS CONCLUSÕES}

O ninho da andorinha-dos-beirais é uma estrutura natural construída com solo argiloso e pode ser utilizado como um modelo biomimético no contexto da construção de terra.

Actualmente, é frequente encontrar ninhos da andorinha-dos-beirais em áreas urbanas e, principalmente, perto dos cursos de água. As dimensões médias de um ninho da andorinha-dos-beirais são $12 \mathrm{~cm}$ de altura,

$18 \mathrm{~cm}$ de largura, $8 \mathrm{~cm}$ de profundidade e 2 $\mathrm{cm}$ de espessura de parede.

O ninho tem uma durabilidade notável atendendo a que é reutilizado em diversos anos.

Uma simulação numérica do ninho permitiu verificar que esta estrutura natural trabalha essencialmente à compressão.

O processo de identificação do tipo de polissacarídeo/açúcar de cromatografia iónica revelou-se muito complexo. Os resultados experimentais obtidos através deste processo, ainda que não sendo totalmente coerentes, indicam que a glicose poderá ser o tipo de polissacarídeo/ açúcar naturalmente adicionado pela andorinha-dos-beirais. Foi sugerido que deverá ser realizado um trabalho de investigação adicional usando um maior número de amostras de solo argiloso da região e também um maior número de amostras de ninho e de forma a dissipar esta possível incoerência.

Os ensaios de compressão mecânicos sugerem que a adição de polissacarídeos/ açúcares no solo argiloso poderá traduzir-se num aumento das propriedades mecânicas de material, em especial um aumento da resistência à compressão. Imagens microscópicas também sugerem que a inclusão destes componentes orgânicos poderá induzir um processo de aglutinação. Estas informações podem ser extrapoladas e utilizadas na melhoria de propriedades de material no contexto da construção de terra.

\section{Bibliografia}

André, A., Pacheco, P., Fonseca, A. (2003). Préesforço orgânico - Estudos sobre a aplicação de uma nova tecnologia. V Simpósio EPUSP sobre estruturas de concreto. São Paulo.

ANSYS - Swanson Analysis Systems Inc. Houston, Version 11.0, 2008.

Bar-Cohen, Y. (2006). Biomimetics, Biological Inspired Technologies. (1. ed). California, EUA: Taylor \& Francis.

Carvalho, J., Pinto, J., Varum, H., Jesus, A., Lousada, J., Morais, J. (2008). Estudo do material terra usado nas construções em tabique na região de Trás-os-Montes e Alto Douro. VII Seminário Ibero-americano de Construção com Terra e II Congresso de Arquitetura e Construção com Terra no Brasil. Universidade Estadual do Maranhão, São Luiz, MA, Brasil.

Lee, Y. C. (1996). Carbohydrate analyses with high-performance anion-exchange chromatography. Journal of Chromatography A, 137-149.

Rael, R. (2008). Earth Architecture. Princeton: PAPress editora. Consultado online no dia 28 de Março de 2009 em http://www. eartharchitecture.org/.

Silva, B., Nunes, F., Pinto, A., Tavares, P., Varum, H., Pinto, J. (2009). Perspectiva biomimética do ninho de andorinha-dos-beirais. Livro de Publicações de Resumos do VIII Seminario Iberoamericano de Construcción com Tierra (VIII SIACOT) e do II Seminario Argentino de Arquitectura y Construcción com Tierra (II SAACT). Tucumán, Argentina: CRIATIAC - FAU - UNT, 100-101. 